\title{
Zufall und Wahrscheinlichkeit - einst ganz getrennt, jetzt eng verbunden
}

\begin{abstract}
Robert Ineichen
Robert Ineichen ist in Luzern aufgewachsen. Er studierte an der Universität Zürich, an der ETH Zürich und an der Sorbonne in Paris. 1950 doktorierte er bei R. Fueter an der Universität Zürich. Nach Lehrtätigkeit am Gymnasium der Kantonsschule Luzern und am Zentralschweizerischen Technikum Luzern, wo er auch des Amt des Vizedirektors versah, wurde er Professor an der Universität Freiburg (Schweiz). Seit 1991 ist er emeritiert. Seine Hauptinteressen liegen im Gebiet der Schul- und Elementarmathematik sowie der Stochastik. In den letzten Jahren wandte er sich vor allem der Geschichte der Mathematik zu. Robert Ineichen ist Verfasser von mehreren Büchern für den Unterricht an Gymnasien und Sekundarschulen.
\end{abstract}

Die Wahrscheinlichkeitsrechnung ist ein Teilgebiet der Mathematik, das sich damit befasst, die Gesetzmässigkeiten unter den zufälligen Ereignissen aufzudecken und zu untersuchen.

Mit dieser Aussage beginnt zum Beispiel Marek Fisz (1970) sein bekanntes Lehrbuch und in ähnlicher Art wird heute in vielen Lehrbüchern der Stochastik einleitend der Zusammenhang von Zufall und Wahrscheinlichkeit betont. Ohne irgendwie Vollständigkeit anzustreben, wollen wir hier der Frage nachgehen, wie die Bereiche des Zufälligen und des Wahrscheinlichen schliesslich zusammengekommen sind.

\section{Pascal: "Mathematik des Glücksspiels" - aleae geometria}

Als Geburtsjahr der Wahrscheinlichkeitsrechnung wird oft das Jahr 1654 genannt, also das Jahr des berühmt gewordenen Briefwechsels zwischen Blaise Pascal (1623-1662)

Die Begriffe Zufall und Wahrscheinlichkeit stehen für uns sehr nahe beieinander; so zögern wir nicht, sogar im Unterricht den einen zu benützen, um den andern zu erklären. Es überrascht zu vernehmen, dass dem nicht immer so war! Robert Ineichen beschreibt die historische Entwicklung der beiden Begriffe, die in vielen Einzelschritten zur heutigen, für uns so selbstverständlichen Situation führte. - Es handelt sich bei diesem Beitrag um die ausgearbeitete Fassung eines Vortrages am Festkolloquium der Universität Münster anlässlich der Emeritierung von Prof. Herbert Kütting am 30. Januar 1998. ust 
und Pierre de Fermat (1601-1665). In ihren Briefen haben die beiden Gelehrten unter anderem bekanntlich das sogenannte Teilungsproblem (le problème des partis) behandelt und auch richtig gelöst. Dieses Problem gehört heute ganz selbstverständlich in die Wahrscheinlichkeitsrechnung. ${ }^{1)}$ Ein einfacher Fall dieses Problems ist der folgende: $A$ und $B$ spielen ein reines Glücksspiel, wobei jeder 32 Goldmünzen einsetzt. Jener, der zuerst drei Partien gewonnen hat, soll den gesamten Einsatz von 64 Goldmünzen erhalten. Nun muss das Spiel abgebrochen werden, nachdem $A$ zwei Partien, $B$ aber nur eine Partie gewonnen hat. Wie haben sie nun die 64 Goldmünzen gerecht zu verteilen? Pascal stellt in seinem Brief an Fermat vom 29. Juli 1654 seine Lösung so dar: Wenn sie noch eine Partie spielen, und dabei $A$ gewinnen würde, so würde $A$ die 64 Goldmünzen erhalten. Wenn aber $B$ diese weitere Partie gewinnen würde, so hätte jeder zwei Partien gewonnen und jetzt würde natürlich jeder seinen Einsatz, nämlich 32 Goldmünzen, zurückerhalten. Dem Spieler $A$ sind also in jedem Fall 32 Goldmünzen sicher. Die anderen 32 Goldmünzen aber wird vielleicht $A$, vielleicht $B$ erhalten. Pascal schreibt: Le hasard [der Zufall] est égal; sinngemäss übersetzt: Die Aussichten dafür sind gleich. Deshalb sollen nach Pascal $A$ und $B$ davon je die Hälfte erhalten. $A$ hat also, wenn diese weitere Partie nicht gespielt wird (s'ils ne veulent point hasarder cette partie), Anrecht auf $32+16=48$ Goldmünzen. $B$ hingegen auf 16 Goldmünzen [17, 24].

Der Mathematiker könnte hier jetzt sofort sagen, dass einfach der Erwartungswert berechnet worden ist; tatsächlich erhält man ja 48 auch aus $(1 / 2) \cdot 64+(1 / 2) \cdot 32$. Doch dies entspricht nicht Pascals Vorgehen: Er arbeitet noch gar nicht mit Wahrscheinlichkeiten, die dann mit Gewinnen zu multiplizieren wären, ${ }^{2)}$ und er verwendet auch nicht jenen Erwartungswert, mit dem später Christiaan Huygens arbeiten wird. Es geht ihm nur um die noch nicht sicher zugeteilten restlichen 32 Goldmünzen, die eben zu halbieren sind, denn Le hasard est égal. Vom Zufall - le hasard - ist also die Rede, doch das Wort "Wahrscheinlichkeit" - la probabilité - kommt in diesem Briefwechsel zwischen Pascal und Fermat nicht vor; es tritt nicht einmal in jenem Brief von Fermat an Pascal auf, wo Fermat mit Quotienten arbeitet, die man heute durchaus als klassisch berechnete Wahrscheinlichkeiten (Zahl der günstigen Fälle durch die Zahl der gleichmöglichen Fälle) auffassen könnte, was O.B. Sheynin zur Feststellung veranlasst: Thus Fermat used the 'classical' definition of probability [26].

Schon viel früher hat auch Girolamo Cardano (1501-1576) in seinem Liber de Ludo Aleae (um 1564 [4]) bei der Beurteilung von Gewinnaussichten im Glückspiel Quotienten aus der Zahl der günstigen und der Zahl der gleichmöglichen Fälle berechnet;

1) Das Teilungsproblem (le problème des partis, the problem of points) ist wohl das wichtigste Problem in der Vor- und Frühgeschichte der Glücksspielrechnung. Es begegnet uns zum ersten Mal in einer Handschrift, die um 1400 entstanden ist; die älteste gedruckte Quelle ist die Summa de Arithmetica Geometria Proportioni et Proportionalita (Venedig 1494) von Luca Pacioli (1445-1517). Siehe z.B. I. Schneider [24, p. 9ff.].

2) Mit solchen Produkten rechnet - wenigstens im Prinzip - Nikolaus Bernoulli (1687-1759) in seiner Dissertation De Usu Artis Conjectandi in Jure von 1709 [2]. Abraham de Moivre gibt die heute übliche Definition des Erwartungswertes [15], [24, p. 105]. 
allerdings ohne diese Fachwörter und ohne das Wort probabilitas zu verwenden. ${ }^{3)}$ Doch er spricht ausdrücklich vom Zufall: Es vermag nämlich in diesen [Dingen] auch der Zufall [fortuna - das Glück] sehr viel. ${ }^{3)}$ - Gehen wir noch weiter zurück: In seiner Divina Commedia erwähnt Dante (1265-1321) den gioco della zara: Ein Spieler wirft drei Würfel, der andere versucht, die Summe der drei Augenzahlen vorauszusagen. Schon in einem frühen Kommentar zur Divina Commedia, in jenem von Jacopo di Giovanni della Lana, geschrieben 1324-28, findet man erste Schritte in Richtung einer stochastischen Betrachtungsweise [13]. Es wird z.B. herausgestellt, dass 3 und 18 jeweils nur auf eine Weise erzeugt werden können, dass aber die Summen von 5 bis 16 auf mehr Arten realisiert werden können, als 3, 4, 17 und 18. Von Wahrscheinlichkeit bzw. probabilità wird auch hier nicht gesprochen, aber vom Zufall wird gesprochen: Oftmals kommt gerade jene Summe, die [eigentlich] weniger oft kommen kann.

In aller Ausführlichkeit und mit stichhaltigen Begründungen wird dieses Problem der drei Würfel zwischen 1250 und 1300 im Pseudoovidius De Vetula ${ }^{4)}$ gelöst: Für jede Summe der drei Augenzahlen wird die Zahl der cadentiae, also der Fälle angegeben, auf dass der Leser die Stärken (virtutes) und die Schwächen (debilitates) der verschiedenen Summen von Augenzahlen erkennen kann. Probabilitas kommt nicht vor, wieder aber wird deutlich auf den Zufall hingewiesen, z.B. mit den Worten: [...] ich aber sage dir [...],dass darin der Zufall [casus] stecken muss, wenn das Glück [sors] dir oder deinem Kameraden den besseren Wurf zugesteht [13, 24].

Teilungsprobleme und viele Probleme über Würfelspiele gehören heute eindeutig zur Wahrscheinlichkeitsrechnung; sie sind aber offenbar lange ohne den Begriff der Wahrscheinlichkeit behandelt worden. Die Frage, wie dann eigentlich die Wahrscheinlichkeit in die Wahrscheinlichkeitsrechnung hineingekommen ist, scheint also berechtigt zu sein. $\mathrm{Zu}$ ihrer Beantwortung ist es notwendig, zuerst einen kurzen Blick in die Antike zu werfen.

\section{Spiele mit Würfeln und Knöcheln in der Antike}

Griechen und Römer haben gewürfelt, zum Teil sogar überaus eifrig. ${ }^{5)}$ Sie haben dazu einerseits die üblichen Würfel verwendet, also mehr oder weniger reguläre Hexaeder, andererseits aber auch Knöchelchen, die sogenannten Astragale. Der Würfel heisst bei den Griechen kybos, bei den Römern tessera. Das lateinische alea kann auch Würfel

3) Cardano schreibt zum Beispiel beim Ausspielen von zwei Würfeln über das Auftreten der Summe 10 ([4], p. 265): Die Summe besteht aus (5,5) und (6,4); letzeres kann aber auf zwei Arten entstehen [hoc enim variatur dupliciter], so dass die Gesamtzahl der Wege, die 10 ergeben, 1/12 der gesamten Möglichkeiten [duodecima pars circuitus] ist. Der zitierte Hinweis über das Wirken des Zufalls lautet bei Cardano: Plurimum valet etiam fortuna in his ([4], p. 264).

4) Der sogenannte Pseudoovidius De Vetula stellt eine fingierte Autobiographie von Ovid dar, die im 13. Jh. entstanden ist. Sie ist in lateinischen Hexametern geschrieben; der Verfasser ist unbekannt.

5) Es gibt vor allem aus römischer Zeit eine grosse Zahl von Zeugnissen, die vor dem Würfeln warnen. Dies zeigt, dass der Würfel im Rom der Kaiserzeit fast allgegenwärtig war. Im Rom der republikanischen und der kaiserlichen Zeit war das Gücksspiel mit Astragalen und Würfeln durch Gesetze (leges aleariae) verboten; die Spielverbote waren nur im Dezember, während der Saturnalien, ausser Kraft. 


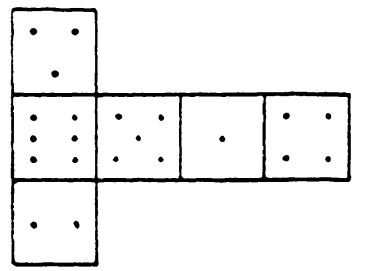

Fig. 1 Würfel waren bereits im Altertum weit verbreitet. - Das Bild zeigt die Abwicklung des wohl ältesten bekannten Würfels, aus Tepe Gawra (Irak). Er ist aus Ton und soll aus dem Beginn des 3. Jahrtausends v. Chr. stammen. [14]
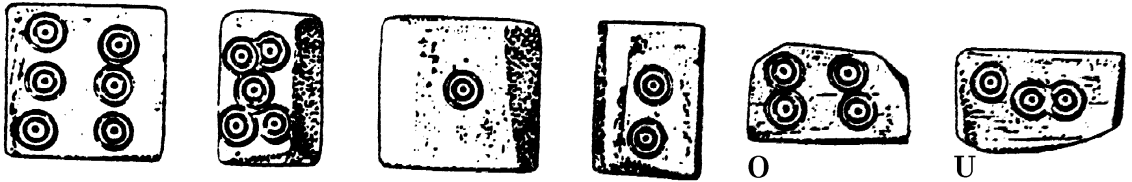

Fig. 2 Mantel, Deck- und Grundfläche $(\mathrm{O} / \mathrm{U})$ eines beinernen Würfels aus dem römischen Legionslager Vindonissa; 1. Jh. n. Chr., Knochen in diesem Fall nicht näher bestimmbar. (Oft sind solche Würfel aus Röhrenknochen des Mittelfusses oder der Mittelhand des Rindes hergestellt worden: Man hat die gewölbten Aussenwände der Knochen abgeschliffen und den erhaltenen vierkantigen Stab in mehr oder weniger würfelförmige Stücke zersägt; die vorhandenen Höhlungen sind mit Knochenzapfen verschlossen worden.) [14]

bedeuten, heisst aber oft auch Wagnis, Zufall, Glücksspiel und Ähnliches. ${ }^{6)}$ Man hat meistens mit drei Würfeln gspielt. Wer die grösste Augensumme erzielte, gewann den gesamten Einsatz: 6, 6, 6 war also der beste Wurf.

Beim Glücksspiel mit dem Astragalos hat man dagegen oft vier Astragale verwendet. Der Astragalos (talus, taxillus) ist ein kleiner Knöchel aus der hinteren Fusswurzel von Schaf oder Ziege. Man könnte ihn als vierseitiges Prisma bezeichnen, dessen vier Seitenflächen aber nicht eben sind und dessen Grund- und Deckfläche gerundet sind. Auf der Grundund der Deckfläche kann der Astragalos nicht stehen; für das Spiel waren nur die vier Seitenflächen massgebend, die sich leicht voneinander unterscheiden lassen und deshalb in der Regel keine "Augen”, Ziffern oder Buchstaben aufwiesen. In gewissen Spielen sind den vier Seitenflächen jedoch Zahlenwerte zugeordnet worden, die man wohl vom Würfel übernommen hat: 1 und 6 für die beiden schmalen, 3 und 4 für die beiden breiten einander gegenüberliegenden Seiten, $[14]^{7)}$.

6) Caesars griechischer Biograph, Plutarch, berichtet, wie Caesar bei der Überschreitung des Rubicon ausgerufen habe anerryphto kybos - Der Würfel sei hochgeworfen. Mit diesem Sprichwort, das auf einen Vers von Menander zurückgeht, wollte er wohl sagen, dass das Wagnis nun eingegangen sei. Die bekannte lateinische Übersetzung iacta alea est geht auf Suetonius zurück; in verbesserter Form lautet sie iacta alea esto.

7) In meinem Buch [14] habe ich versucht, Zeugnisse zusammenzustellen und zu kommentieren, in denen Begriffe und Überlegungen auftreten, die ins Umfeld von Begriffen und Überlegungen gehören, die auch in unserem heutigen stochastischen Denken vorhanden sind. Auf dieses Buch sei auch für alle weiteren Literaturangaben im Zusammenhang mit der Antike verwiesen. 

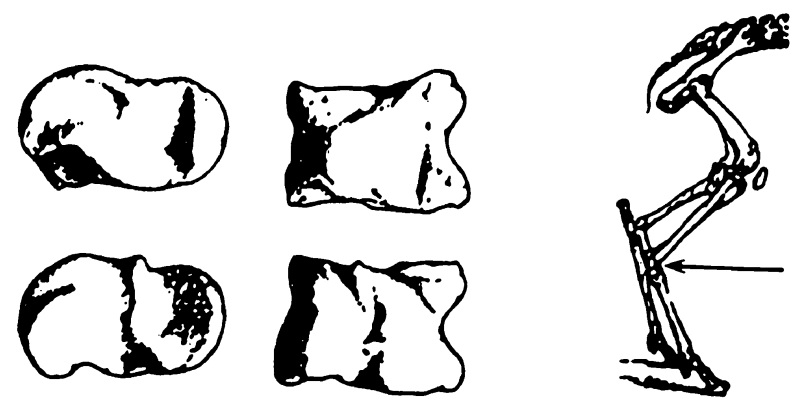

Fig. 3 Astragale. Jeder der vier Astragale zeigt eine andere Seitenfläche. Rechts die Hinterbeine eines Schafes. Der Pfeil bezeichnet die Lage des Astragalos ( $\alpha \sigma \tau \rho \alpha ́ \gamma \alpha \lambda \lambda_{\circ}$, talus, Sprungbein) in der Fusswurzel. [14]

Beim Spiel mit den vier Astragalen galt der Venuswurf (iactus Venerius, Aphrodite-Wurf) als bester Wurf: Jeder Astragalos zeigte dann eine andere Seite; der schlechteste Wurf war der Hunde-Wurf (canis; kyon): vielleicht ein Wurf mit viermal der Seite 1. Es sind 35 Seitenkombinationen möglich: 35 Kombinationen mit Wiederholung der Länge vier aus vier Elementen (aus den vier Seitenflächen). Es sind nur wenige Namen solcher Seitenkombinationen überliefert; noch weniger weiss man über die Werte der einzelnen Seitenkombinationen. Man kann aber immerhin feststellen, dass man nicht einfach die oben erwähnten Zahlenwerte addiert hat. Die Bewertung der einzelnen Kombinationen muss kompliziert gewesen sein: Ovid berichtet von Autoren, die Bücher zum Thema quid valeant tali - was die Knöchel gelten sollen - geschrieben haben.

Eine andere Form des Spieles mit den Astragalen ist das Meistwurfspiel (pleistobolinda): Jeder der beiden Spieler startet mit gleich vielen Astragalen. Jeder wirft jeweils einen Astragalos. Jener Spieler, dessen Astragalos den höheren Wert zeigt, behält seinen Knöchel und gewinnt dazu den vom Gegner ausgeworfenen Knöchel. Dabei kann der zeitweilig Unterlegene natürlich auch wieder gewinnen, bis der Kampf schliesslich nach aufregendem Hin und Her entschieden ist, weil ein Spieler alle Knöchel verloren hat. Diese Form des Spieles könnte gemeint sein, wenn Homer im 23. Gesang der llias erzählt, wie Achilleus im Schlaf von des Patroklos Seele besucht und daran erinnert wurde, wie sie schon in der Jugend vereint gewesen (87-88):

Damals als ich den Sohn des Amphidamas hatte getötet,

unbedacht gegen mein Wollen, aus Zorn beim Spiele der Knöchel.

Hat man in der Antike mit dem Würfel oder beim Astragalspiel etwas von der statistischen Regelmässigkeit, von der Stabilität der relativen Häufigkeiten, wahrgenommen? Davon ist uns gar nichts überliefert, was eigentlich erstaunlich ist. Wir dürfen nur aus vielen Zeugnissen schliessen, dass in der Antike mit den Astragalen und mit den Würfeln wirklich auch Glücksspiele, also Zufallsexperimente, durchgeführt worden sind, dass diese Objekte nicht nur bei Orakeln oder zur Divination verwendet worden sind: Geratewohl und Zufall - temeritas et casus - wirken, schreibt Cicero [14]. Wir wissen aber weiter, dass es auch mit Blei beschwerte Astragale gegeben hat, ferner Astragale mit angeschliffenen Seiten und Würfel, die offensichtlich für das Falschspiel präpariert worden 
sind: Durch Kunst Fortunas Fehler wieder gut zu machen - ut arte emendaturus fortuna (Horaz, [14]). Darf man daraus nicht schliessen, dass man andere, bessere Ergebnisse erzielen wollte, dass man diesen Astragalen und Würfeln eine gewisse Tendenz aufzwingen wollte, dass man also relative Häufigkeiten eben doch gelegentlich auch beachtet hat? Aber Schätzwerte für Wahrscheinlichkeiten haben Römer und Griechen weder für die Seiten des Astragalos, ${ }^{8)}$ noch für ihre Ergebnisse beim Würfeln bestimmt; überhaupt haben sie ihren Wahrscheinlichkeitsbegriff, auf den wir noch zu sprechen kommen, im Zusammenhang mit den Glücksspielen nicht verwendet. Es muss auch gesagt werden, dass der beste Wurf mit den vier Astragalen, der Venuswurf, keineswegs die seltenste Seitenkombination, also jene mit der kleinsten Wahrscheinlichkeit ${ }^{8}$, gewesen ist. Man könnte sich hingegen denken, dass der Venuswurf vielleicht deshalb als bester Wurf betrachtet worden ist, weil er der ausgeglichenste ist, weil er eine gewisse Harmonie verkörpert, was ja nach unserem Verständnis der Antike etwas sehr Erstrebenswertes ist.

Es gab in der Antike auch Listen mit den 216 gleichmöglichen Fällen, die auftreten können, wenn drei gute Würfel geworfen werden. Doch man hat keine Zeugnisse, dass solche Listen verwendet worden sind, um zu erwägen, mit welcher Leichtigkeit die eine oder andere Kombination auftreten könnte. Das Prinzip des mangelnden Grundes, wie es noch Laplace verwendet, um die gleichmöglichen Fälle zu definieren, ${ }^{9)}$ wäre beim Philosophen Anaximander (610-547) zu finden gewesen, allerdings nicht in einem stochastischen Zusammenhang. S. Sambursky [20] hat darauf hingewiesen, dass die Stoiker eigentlich bis an die Schwelle der Wahrscheinlichkeitslehre gekommen sind: Sie betrachteten potentielle Geschehnisse, die im Rahmen des Fatums gleichmöglich sind, und zudem haben sie ja auch die Lehre von den Disjunktionen ausgearbeitet. Doch sie haben die Anfänge einer Wahrscheinlichkeitsrechnung nicht entwickelt. ${ }^{7)}$

\section{3 Über den Wahrscheinlichkeitsbegriff in der Antike}

Beachten wir zunächst, dass in unserer Umgangssprache das Wort "wahrscheinlich" besagt, das die Gründe für die Geltung einer Aussage oder für das Eintreffen eines Ereignisses zwar überwiegen, aber doch nicht ausreichen, um das Gegenteil auszuschliessen; in der Stochastik würde man in dieser Situation etwa sagen: mit grosser Wahrscheinlichkeit. In der Literatur zu den Grundlagen der Stochastik stösst man auf verschiedene Arten von Wahrscheinlichkeit. Am meisten verbreitet ist heute die Unterscheidung von zwei Aspekten des Wahrscheinlichkeitsbegriffs, anders gesagt, von zwei Arten von Wahrscheinlichkeit, nämlich der aleatorischen Wahrscheinlichkeit und der epistemischen Wahrscheinlichkeit; allerdings eine Unterscheidung, die man in der Antike noch nicht gekannt hat.

8) Als Schätzwerte erhält man für die Wahrscheinlichkeiten der Seiten 1 und 6 je ungefähr $10 \%$, für die Seiten 3 und 4 je ungefähr $40 \%$. - Damit ergibt sich für die Wahrscheinlichkeit des Venuswurfes ein Schätzwert von ungefähr $4 \%$. Vgl. [14].

9) Für P.S. de Laplace sind in seinem Essay Philosophique sur les Probabilités von 1814 gleichmögliche Fälle solche, über deren Existenz wir in gleicher Weise unschlüssig sind - également indécis sur leur existence. 
Der aleatorische Aspekt des Wahrscheinlichkeitsbegriffs, kurz also die aleatorische Wahrscheinlichkeit, tritt uns besonders deutlich entgegen, wenn es um die Wahrscheinlichkeit bei Glücksspielen oder bei ähnlich gelagerten Zufallsexperimenten geht oder bei Ziehungen aus Populationen; die aleatorische Wahrscheinlichkeit ist die Wahrscheinlichkeit eines zufälligen Ereignisses. Heute wird sie durch numerische Werte ausgedrückt, die man entweder a priori durch den Quotienten aus der Zahl der günstigen Fälle und der gesamten Zahl der gleichmöglichen Fälle gewinnt oder von der man a posteriori aus genügend vielen Versuchen Schätzwerte bestimmt. Die aleatorische Wahrscheinlichkeit ist also eigentlich in der Sache begründet. Man nennt sie oft auch objektive ${ }^{10)}$ oder auch statistische Wahrscheinlichkeit.

Der epistemische Aspekt des Wahrscheinlichkeitsbegriffs, kurz also die epistemische Wahrscheinlichkeit, drückt den Grad des Vertrauens in eine Aussage aus, den Grad ihrer Glaubwürdigkeit. Diese epistemische Wahrscheinlichkeit können wir oft auch bei Behauptungen finden, die (eventuell) gar keinen statistischen Hintergrund haben; sie ist im unvollständigen Wissen um eine Sache, in den unvollständigen Kenntnissen über einen behaupteten Sachverhalt, begründet. Sie wird oft auch als subjektive ${ }^{10)}$ oder persönliche Wahrscheinlichkeit bezeichnet. Auch hier können numerische Werte für den Grad des Vertrauens gefunden werden, auf verschiedene Weisen, so zum Beispiel durch Wetten. ${ }^{11}$ ) Bekanntlich gehen verschiedene Forscher, die sich mit den Grundlagen der Wahrscheinlichkeitsrechnung befasst haben, von diesem etwas weiteren, allgemeineren Begriff der Wahrscheinlichkeit aus (z.B. P. Ramsey [18], B. de Finetti [8]). Man kann oder könnte in einer gewissen Hinsicht auch den aleatorischen Aspekt in diesen umfassenderen Begriff einordnen. Als Ausgangspunkt der üblichen Wahrscheinlichkeitsrechnung kommen beide Aspekte der Wahrscheinlichkeit in Frage (vgl. z.B. J.L. Hodges und E.L. Lehmann [11]). Oft ist man heute so stark auf die aleatorische Wahrscheinlichkeit ausgerichtet, dass man den epistemischen Aspekt fast ganz verliert. Dies liegt natürlich sehr nahe, denn man verwendet ja meistens bei der Motivation und bei der Interpretation der Axiome relative Häufigkeiten. Doch kommt eigentlich selbst ein ausgesprochener Frequentist kaum um den epistemischen Aspekt der Wahrscheinlichkeit herum, wenn er z.B. in der mathematischen Statistik im Zusammenhang mit der Berechnung eines Vertrauensintervalls der Vertrauenswahrscheinlichkeit oder beim Testen der Wahrscheinlichkeit des Fehlers 1. Art oder 2. Art begegnet. Hier geht es doch wesentlich um den Grad des Vertrauens, und eine Betrachtung nur unter dem aleatorischen Aspekt - an sich ist dies ja möglich - wirkt doch meistens ziemlich gekünstelt. ${ }^{12)}$ Und wie ist es, wenn die Nasa im Herbst 1997 die Wahrscheinlichkeit, dass die mit Plutonium bestückte Cassini-Sonde in der

10) Die Attribute "objektiv" und "subjektiv" sind nicht sehr geeignet, um die beiden Arten von Wahrscheinlichkeiten zu unterscheiden, denn auch die "objektive Wahrscheinlichkeit" enthält ein gewisses subjektives Element: Es muss ja ein Modell gewählt werden, um eine aleatorische Wahrscheinlichkeit zu bestimmen, und so kommt unweigerlich auch ein subjektiver Einfluss hinein.

11) Es gibt verschiedene Arbeiten zu den Grundlagen der Wahrscheinlichkeitsrechnung, die einen Wettbegriff verwenden. - Interessant ist, dass bereits Immanuel Kant in seiner Kritik der Reinen Vernunft schreibt: Der gewöhnliche Probierstein, ob etwas blosse Überredung oder wenigstens subjektive Überzeugung, d.i. festes Glauben, sei, was jemand behauptet, ist das Wetten.

12) Das Gesetz der grossen Zahlen von Jakob Bernoulli sagt uns bekanntlich im wesentlichen, dass die relative Häufigkeit eines zufälligen Ereignisses bei einer grossen Zahl Versuchen sehr wahrscheinlich nahe bei 
Erdatmosphäre verglüht, mit eins zu einer Million beziffert? Wollen wir nicht lieber in der Wahrscheinlichkeit von 999999 Millionsteln, dass dieses Verglühen nicht geschieht, einen sehr hohen Grad des Vertrauens sehen, den wir der beruhigenden Nasa-Aussage zubilligen, anstatt sie als aleatorische Wahrscheinlichkeit aufzufassen?

Bereits in Dialogen von Platon (427-347) finden wir nun oft den epistemischen Wahrscheinlichkeitsbegriff, um die hohe Glaubwürdigkeit, die starke Plausibilität, auszudrücken. Das griechische Äquivalent dafür ist eikos. Platon stellt auch die bloss wahrscheinliche Begründung einer Aussage dem zwingenden Beweis der Mathematiker gegenüber. Aristoteles (384-322) definiert in der Topik: Wahrscheinliche Sätze sind diejenigen, die allen oder den meisten oder den Weisen als wahr erscheinen, und auch von den Weisen wieder entweder allen oder den meisten oder den bekanntesten und angesehensten. Hier ist es das griechische endoxos, das durch wahrscheinlich übersetzt wird. Wieder geht es um die epistemische Wahrscheinlichkeit von Aussagen. Weiter tritt bei Aristoteles das Wort pithanos in der Bedeutung von wahrscheinlich, glaubwürdig auf; eikos kommt ebenfalls wieder vor, so zur Bezeichnung von dem, was sich meistens ereignet. Dieses "meistens", das hier auftritt, zeigt uns, dass ein leiser aleatorischer Aspekt auch mit dem epistemischen verbunden auftreten kann: eine grosse relative Häufigkeit gestattet eben auch eine Aussage von hoher Glaubwürdigkeit. In Ciceros philosophischen Schriften werden die genannten griechischen Fachausdrücke durch das Wort probabilis ins Lateinische übersetzt; auch bei ihm klingt gelegentlich der aleatorische Aspekt an, allerdings nur schwach. ${ }^{7)}$

Besonders zu beachten ist aber jetzt, dass sich Aristoteles in seiner Physik und in seiner Metaphysik mit dem Begriff des Zufalls auseinandersetzt; er ist ja der erste Philosoph, von dem uns ausführliche Überlegungen zum Zufallsbegriff zur Verfügung stehen. To automaton (das "Vonselbst") nennt er den Zufall; von tyche spricht er in jenem Spezialfall, wo etwas unbeabsichtigt aus einer beabsichtigten Handlung entsteht. Ganz wesentlich ist nun in unserem Zusammenhang eine Aussage, die sich in seiner Metaphysik und ähnlich auch in anderen seiner Schriften findet: Ursachen aber, durch welche das Zufällige geschehen kann, sind unbestimmt; darum ist er [der Zufall] für menschliche Überlegungen unerkennbar. Das heisst doch auch, dass ein Bemühen, diesen Zufall durch Abwägen von Chancen, modern gesprochen, durch Abschätzen von Wahrscheinlichkeiten, in etwa in den Griff zu bekommen, von vorneherein zum Scheitern verurteilt wäre: Das Wahrscheinliche und das Zufällige sind getrennt! Und so kommt J. van Brakel [3] zusammenfassend zu folgender Feststellung, der auch viele andere Autoren zustimmen: Very roughly, for the 15 centuries from Aristotle to Thomas [Thomas von Aquino, 122574], the picture is, that things can be distinguished in three classes: (i) that of which certain knowledge is possible, (ii) that of which probable knowledge is possible, and (iii) that of which no knowledge is possible. Nach Platon entsprechen die zwei ersten Kategorien dem Wissen (scientia) bzw. der Meinung (opinio). Die dritte Kategorie meint den Bereich des Zufälligen; darüber glaubte man, keine weiteren Erkenntnisse gewinnen zu können. - "Wahrscheinlich", probabilis, ist also in der Antike Attribut einer Meinung

der zugehörigen (aleatorischen) Wahrscheinlichkeit dieses Ereignisses liegt. Aber dieses "wahrscheinlich" meint doch eigentlich eine epistemische Wahrscheinlichkeit! G. Shafer macht in [25] deutlich auf diese Sachlage aufmerksam. 
und sagt etwas aus über die Glaubwürdigkeit der ausgesagten Meinung. Die epistemische Wahrscheinlichkeit, wie sie für die griechisch-römische Antike typisch ist, ist getrennt vom Bereich des Zufälligen! ${ }^{13)}$

Numerische Werte für solche epistemische Wahrscheinlichkeiten finden wir in der Antike nicht; nicht einmal bei den Hippokratikern, die doch ausdrücklich die Berücksichtigung von praktischen Erfahrungen fordern. Aber immerhin findet man im Corpus Hippocraticum gelegentlich Aussagen, die eine erste Graduierung des Wahrscheinlichen zeigen: Es werden beispielsweise - rein qualitativ - Mortalitäten von einzelnen Krankheiten in verschiedenen Jahreszeiten verglichen; oder es wird darauf hingewiesen, dass bei gewissen Unfällen das Risiko des Sterbens grösser ist als bei anderen. Auf eine eigentliche Graduierung stösst man dann bei Karneades (213-129), und ähnlich auch später beim römischen Rhetoriker Quintilianus (1. Jh. n. Chr.). Karneades unterscheidet bei dem als wahr Erscheinenden - beim phitanon, beim probabile - drei Stufen: wahrscheinliche Vorstellungen - wahrscheinliche und unbestrittene Vorstellungen - schliesslich wahrscheinliche, unbestrittene und allseitig geprüfte Vorstellungen. ${ }^{7)}$

\section{Zufall und Wahrscheinlichkeit - wie kamen sie schliesslich zusammen?}

Dieser sehr interessanten Frage sind verschiedene Forscher nachgegangen, so z.B. E. Coumet [5], L. Daston [6], G. Gigerenzer e.a. [9], J. Hacking [10], G. Shafer [25] und vor allem immer wieder I. Schneider [21, 22, 23, 24 usw.]. I. Schneider hat sehr klar herausgearbeitet, was es gebraucht hat, damit die Wahrscheinlichkeitsrechnung entstehen konnte, deren Grundlagen dann schliesslich Jakob Bernoulli geschaffen hat: Zwei voneinander unabhängige Entwicklungen führten durch ihr Zusammentreffen zur Mathematisierung des Wahrscheinlichen in der Zeit zwischen Pascal und de Moivre. Es handelt sich dabei um die Wandlung des Bedeutungsinhaltes von probabilitas zu einem quantifizierbaren Begriff und zum anderen um das Konzept des Zufalls, das im Rahmen der Glücksspielrechnung in einer Chancenverhältnisrechnung mathematisiert wurde [21]. Es ist nicht möglich, hier auf wenig Raum die Entwicklung dieser beiden Ströme und ihr Zusammenfliessen vollständig zu schildern; es sollen aber skizzenhaft einige Hinweise gemacht werden.

Gehen wir zuerst dem zweiten Strom etwas nach, der Chancenverhältnisrechnung bei den Glücksspielen: Sowohl im Mittelalter wie in der frühen Neuzeit haben massgebende Theologen immer wieder die Glücksspiele um Geld bekämpft. Noch 1619 stellt der hl. Franz von Sales fest, dass ein Gewinn doch der Preis des Fleisses sein sollte, aber beim Glücksspiel sei er der Preis des Zufalls [des Geschicks], der eigentlich keinen Preis verdiene, denn er hänge keineswegs von uns ab [19]. Wir haben bereits oben gesehen, dass trotzdem immer wieder gespielt worden ist, und das ebenfalls bereits erwähnte Buch von Girolamo Cardano, der Liber de Ludo Aleae (um 1564), stellt übrigens viel eher ein Anleitung für Glücksspieler dar als ein Buch für Mathematiker. Bei dieser Sachlage ist es eigentlich nicht verwunderlich, dass gerade in Kreisen, die sowohl der Scholastik - wo ja immer noch Aristoteles nachwirkte - wie auch der Theologie eher fernstanden, auch eine Mathematisierung und Quantifizierung des Zufälligen, wie es

13) Probabilis hat allerdings noch viele weiter Bedeutungen (siehe Th. Deman [7]). 
sich eben in Glücksspielen zeigt, versucht worden ist: Die vor allem in Handeslkreisen grassierenden Glücksspiele wurden als eine zeitlich verkürzte Form der Tätigkeit des Kaufmanns verstanden. In den für den Kaufmann verfassten Rechenbüchern tauchen ab dem Ende des 15. Jahrhunderts und im 16. Jahrhundert die ersten Versuche auf, Glücksspielprobleme rechnerisch zu lösen (I. Schneider [22]).

Ausserdem setzte sich nun in weiten Kreisen immer mehr die Auffassung durch, dass es auch nach dem Naturrecht erlaubt sei, eine Ware oder Geld dem Zufall auszusetzen, indem man einen vorhandenen, gegenwärtigen Wert gegen einen ungewissen zukünftigen Wert einsetzte und dabei eine Abmachung machte von der Form des Do ut des: Man kauft z.B. die Getreideernte des kommenden Jahres und zahlt dafür jetzt - der ungewisse, zukünftige Wert besteht in der Getreideernte des nächsten Jahres, die man erhalten wird; oder man kauft jetzt eine Leibrente - der ungewisse, zukünftige Wert besteht in den Renten, die man in den kommenden Lebensjahren beziehen wird. Aber ebenso kann man nun auch den Einsatz bei einem Glücksspiel betrachten, den man gegen einen ungewissen, zukünftigen Gewinn einzahlt. In diesen und vielen ähnlich gelagerten Fällen ist das Risiko das moralische Äquivalent für die Arbeit (L. Daston in [6]): Das Spekulieren, also die Übernahme eines Risikos, ist in allen diesen Fällen ebenso ehrenhaft wie die Arbeitsleistung des Handwerkers oder des Bauern.

Die Mathematiker haben das Verhältnis zwischen der Gefahr eines allfälligen Verlustes und der Aussicht auf einen allfälligen Gewinn zu quantifizieren versucht. Bekanntlich hat Christiaan Huygens (1629-1695), der in Paris vom Briefwechsel zwischen Pascal und Fermat gehört hatte, 1656 eine kleine streng systematisch aufgebaute Abhandlung über Glücksspiele geschrieben: Van Rekeningh in Spelen van Geluck. Sie ist durch Frans van Schooten ins Lateinische übersetzt worden und von diesem unter dem Titel De Ratiociniis in Ludo Aleae 1657 in den Exercitationes Mathematicae publiziert worden; 1660 ist die Abhandlung von Huygens dann auch in Holländisch erschienen. Huygens arbeitet hier mit dem Erwartungswert (holländisch het is my soo veel weerdt; lateinisch valor expectationis). Doch wird dieser Erwartungswert nicht etwa durch Produkte von Wahrscheinlichkeiten mit dem zugehörigen Gewinn oder Verlust definiert. Er erklärt ihn durch ein gerechtes, ein unparteiisches, ein rechtmässiges Spiel - rechtmatigh spel. Dies ist also ein Spiel, das nicht darauf zielt, jemandem zu schaden - dat is, daer niemandt verlies geboden werdt $[12, \mathrm{p} .60] .{ }^{14)}$ Da wir heute in der Regel gerechtes Spiel über die Erwartungswerte definieren, scheint uns dieses Vorgehen von Huygens zirkelhaft. Das war es indessen für Huygens keineswegs: Seiner Generation war der Begriff des gerechten, des unparteiischen Spiels intuitiv klar. Die Basis für diese intuitive Klarheit ist in den oben skizzierten Abmachungen begründet, die den Austausch eines gegenwärtigen, eines vorhandenen Wertes gegen einen ungewissen, zukünftigen Wert ermöglicht haben $[5,6]$.

Als Jakob Bernoulli (1655-1705) die lateinische Abhandlung von Huygens las, fand er darin keinen Ausdruck für Wahrscheinlichkeit, sondern den Fachausdruck "valor expec-

14) Es muss hier bemerkt werden, dass in der holländischen Fassung der Schrift von Huygens das Wort kans vorkommt und zwar nach B.L. van der Waerden [2] in zwei Bedeutungen: einerseits als Möglichkeit, etwas zu gewinnen, wie etwa unsere Chance, wenn wir z.B. von der Anzahl der Chancen sprechen andererseits auch in der Bedeutung von Wahrscheinlichkeit. 
tationis", auf den unser heutiges Fachwort "Erwartungswert" zurückgehen dürfte. Dieser Erwartungswert wird z.B. im Satz III der Abhandlung von Huygens so berechnet: Wenn die Anzahl der Fälle, in welchem mir a zufällt, gleich $p$ und die Anzahl der Fälle, in welchen mir $b$ zufällt, gleich q ist, wird meine Erwartung unter der Annahme, dass alle Fälle gleich leicht eintreten können, $(p a+q b) /(p+q)$ sein [12]. Auch Bernoulli arbeitet in seiner Ars Conjectandi [2] über weite Strecken mit solchen Erwartungswerten. Es ist aber nun bemerkenswert, dass sich daraus bei ihm an mancher Stelle Terme der Form $p /(p+q)$ ergeben. Das ist doch bereits der später als "klassische Wahrscheinlichkeit" bezeichnete Ausdruck, der Quotient aus der Anzahl der günstigen Fälle durch die Anzahl der gleichmöglichen! Allerdings spricht Bernoulli hier in der Regel von sors (wohl in der Bedeutung von Geschick, Chance), gelegentlich auch von expectatio. Erst im vierten Teil der Ars Conjectandi führt er dann probabilitas, also Wahrscheinlichkeit, ein. Darauf kommen wir nun gleich noch zurück.

Nun sollen zum anderen der beiden Ströme, also zur Wandlung des Bedeutungsinhaltes von probabilitas zu einem quantifizierbaren Ausdruck, noch kurz einige skizzenhafte Hinweise gemacht werden. Wir haben bereits darauf hingewiesen: In der Antike war probabilis in der Regel Attribut einer Meinung (opinio), die durch eine Autorität garantiert war; dies war auch im Mittelalter und in der Renaissance nicht anders. Dieser Wahrscheinlichkeitsbegriff war qualitativ; immerhin gab es - vor allem seit Karneades gewisse Graduierungen. Bis etwa um 1660 haben aber jene Gelehrten, die sich mit probabilitas beschäftigt haben, nur ganz selten Zusammenhänge zwischen ihrer probabilitas und dem Zufälligen wahrgenommen. Im frühen 17. Jahrhundert jedoch hat nun dieser Wahrscheinlichkeitsbegriff gewisse Veränderungen erfahren. Man begann, verschiedene Stufen der Gewissheit zu unterscheiden: Die durch einen eigentlichen Beweis sichergestellte mathematische Gewissheit als höchste Stufe, etwas tiefer dann die physikalische Gewissheit, beruhend auf der Wahrnehmung der Evidenz, die durch die Sinne gegeben war, und schliesslich die moralische Gewissheit, die durch Zeugnisse und Vermutungen abgestützt war. Im Zusammenhang damit veränderte sich auch der Bedeutungsinhalt von probabilitas: Sie wurde immer mehr zu einem Grad der Zustimmung entsprechend der Evidenz, der Einsichtigkeit, die durch eine Sache oder durch Zeugnisse vorgegeben war [6].

In diesem Kontext ist nun auch Gotfried Wilhelm Leibniz (1646-1716) zu nennen, der sich intensiv mit verschiedenen Aspekten des Wahrscheinlichkeitsbegriffs beschäftigte, dabei auch Möglichkeiten zur Schätzung von Wahrscheinlichkeiten behandelte und hier auch die Konzepte der Antike (Aristoteles, Karneades) in Betracht zog. Lebenslang hegte er, vor allem begründet durch die Bedürfnisse der Jurisprudenz, die Idee, all diese Aspekte in einer Doctrina de gradibus probabilitatis zusammenzufassen (I. Schneider [24, p. 48]). Übrigens bewerteten damals Rechtsgelehrte z.B. Zeugenaussagen gelegentlich auch numerisch: So galt etwa in gewissen Fällen das Zeugnis eines Verwandten des Angeklagten 1/3 von dem, was ein unabhängiges, tadelloses Zeugnis galt (G. Gigerenzer e.a. in [9]).

Eine besonders wichtige Rolle spielte in der Entwicklung dieser Wandlung des Bedeutungsinhaltes von probabilitas die 1662 erschienene 'Logik von Port Royal' von Antoine 
Arnauld und Pierre Nicole, an der auch Pascal beteiligt war, La Logique ou l'Art de Penser [1]. Ihre lateinische Ausgabe ist unter dem Titel Ars Cogitandi erschienen. Jakob Bernoulli war mit diesem weit verbreiteten Werk vertraut; dessen Titel hat ihn vermutlich auch zum Titel Ars Conjectandi für sein Hauptwerk angeregt. Im 16. Kapitel dieser 'Logik von Port Royal' wird nun ausgeführt, dass dort, wo es darum geht, allenfalls zu gewinnen oder zu verlieren - allgemeiner: ein Gut zu erhalten oder ein Übel erleiden zu müssen, - nicht nur Gewinn und Verlust an sich betrachtet werden dürfen: Bei der Beurteilung der Lage ist auch die Anzahl der Grade der Wahrscheinlichkeit (degrés de probabilité) zu berücksichtigen. Um dies zu veranschaulichen, wird ein Glücksspiel betrachtet, bei welchem man neun Taler gewinnen oder einen Taler verlieren kann; doch es ist dabei neunmal wahrscheinlicher (neuf fois plus probable) zu verlieren als zu gewinnen, weil das betrachtete Spiel so eingerichtet ist, dass für den Verlust neun Grade der Wahrscheinlichkeit vorhanden sind, für den Gewinn nur einer. G. Shafer [25] sucht nach einem Grund dafür, dass Antoine Arnauld hier probabilitas und Zufall (durch ein Glücksspiel dargestellt) zusammenbringt: Beim herangezogenen Glücksspiel sind die Gewinn- und Verlustaussichten a priori bekannt, und sie gehören damit auch zu unserer (unvollständigen) Kenntnis der Sache; damit ist aber in diesem Glücksspiel ebenfalls ein epistemischer Aspekt vorhanden, und dieser führt uns zur probabilitas.

Nicht erst in seiner Ars Conjectandi, sondern bereits in seinem mathematischen Tagebuch, den Meditationes [2] aus den achtziger Jahren, findet man Eintragungen, die zeigen, wie Bernoulli sich bemüht, das Glücksspielmodell auf neue Bereiche anzuwenden. Und im IV. Teil der Ars Conjectandi trifft man schliesslich auf die ersten wirklich substantiellen Beiträge zur Verbindung der Glücksspielrechnung mit der probabilitas: Jacob Bernoulli was the first substantial contributor to the theory of games of chances to grapple with its connection with probability (G. Shafer in [25]). In diesem IV. Teil der Ars Conjectandi geht es Bernoulli zunächst um das Vermuten (conjicere) und um die Beurteilung der Beweisgründe für eine Vermutung (argumenta conjecturarum) und dann natürlich um den Lehrsatz, den man später als das Bernoullische Gesetz der grossen Zahlen bezeichnen wird, worauf wir allerdings in diesem Rahmen nicht eingehen können. Eine Sache vermuten heisst, ihre Wahrscheinlichkeit messen. Und diese Wahrscheinlichkeit ist bei Bernoulli ein Grad der Gewissheit und unterscheidet sich von ihr wie ein Teil vom Ganzen. Um diese Wahrscheinlichkeit messen zu können, hat man zuerst die Anzahl und das Gewicht der Beweisgründe zu schätzen. Berechnet werden die Wahrscheinlichkeiten dann nach den Methoden der Glücksspielrechnung. Also einerseits eine klare Trennung: Die Wahrscheinlichkeit als Grad der Gewissheit, als Mass unserer unvollständigen Kenntnisse von einer Sache ist - nach heutiger Bezeichnungsweise - eine epistemische Wahrscheinlichkeit; die Berechnung dieser Wahrscheinlichkeit geschieht nach den Methoden der Glücksspielrechnung, bei der ja jeweils der aleatorische Aspekt im Vordergrund steht. Andererseits aber auch eine oft sehr fruchtbare Verbindung: Wahrscheinlichkeiten, probabilitates, werden nun berechenbar; es ist dies eine Verbindung, die durch die oben skizzierte Entwicklung vorbereitet war. Weiter ist hier zu beachten, dass damals offenbar kein Graben zwischen epistemischer und aleatorischer Wahrscheinlichkeit vorhanden war, wie er heute aus philosphischer Sicht bestehen mag (G. Gigerenzer e.a. [9]). 
Die vielleicht etwas zu scharfsinnigen Unterscheidungen von Bernoulli wurden, wie z.B. G. Shafer [25] betont, von seinen Nachfolgern nicht genau beachtet: Es schien ihnen ganz natürlich, probabilitas zu identifizieren mit dem, was bei den Glücksspielen, wo ja der Zufall mitspielt, als ease of happening (Leichtigkeit des Eintreffens) in Erscheinung tritt. Der Essay d'Analyse sur les Jeux de Hasard [16] von Pierre Rémond de Montmort (1678-1719) erschien 1708. Damals lag die Ars Conjectandi noch nicht gedruckt vor, denn sie erschien erst postum 1713; doch man wusste um sie, man sprach von ihr. Montmort behandelt die Glücksspiele noch zum grossen Teil nach dem Vokabular von Huygens, d.h. er zählt Fälle und berechnet Erwartungswerte; gelegentlich verwendet er in etwas unbestimmter Art das Wort "probabilitê". Der erste, der seine Darstellung nun ausdrücklich auf dem Begriff der probability, also der Wahrscheinlichkeit, aufbaut und sie nach der 'klassischen' Berechnungsart bestimmt, ist Abraham de Moivre (16671754). In De Mensura Sortis (1712) und dann in seinem 1718 erstmals erschienenen Buch The Doctrine of Chances bereitet er die Formulierung vor, die man dann in der 2. Auflage von 1738 [15] lesen kann: Wherefore if we constitue a Fraction where the Numerator be the Number of Chances whereby an Event may happen, and the Denominator the number of all Chances, whereby it may either happen or fail, that Fraction will be a proper designation of the Probability of happening. - 1818 erscheint schliesslich zum ersten Mal das Fachwort "Wahrscheinlichkeitsrechnung": E.S. Unger (1789-1870) übertrug den Calcul des Probabilités von S.F. Lacroix (1765-1843) in die deutsche Sprache und gab ihm den Titel Lehrbuch der Wahrscheinlichkeitsrechnung. Und damit war die Wahrscheinlichkeit endgültig in die Wahrscheinlichkeitsrechnung hineingekommen!

Nur S.D. Poisson (1837) und A.A. Cournot (1843) haben dann wieder Chance (aleatorische Wahrscheinlichkeit) und Probabilité (epistemische Wahrscheinlichkeit) unterschieden. Und selbstverständlich haben später die Untersuchungen der Grundlagen der Wahrscheinlichkeitsrechnung wieder zu verschiedenen Aspekten des Wahrscheinlichkeitsbegriffes geführt.

\section{Literatur}

[1] A. Arnauld, P. Nicole, (1662 bzw. 1965): La Logique ou l'Art de penser. Ed. critique par P. Clair et F. Gibral. Paris: Presses universitaires de France.

[2] J. Bernoulli, (1713 bzw. 1899): Wahrscheinlichkeitsrechnung (Ars conjectandi), übersetzt von R. Haussner. Ostwalds Klassiker der exakten Wissenschaften Nr. 107 und 108. Leipzig: Akadem. Verlagsanstalt. - Bernoulli, J. (1713 bzw. 1975): Die Werke von Jakob Bernoulli, Bd. 3. Bearbeitet von B.L. van der Waerden, Basel: Birkhäuser.

[3] J. van Brakel, (1976): Some remarks on the Prehistory of the Concept of Statistical Probability. Archive for History of Exact Sciences, 16, pp. 119-136.

[4] G. Cardano, (1564 bzw. 1663): Liber de ludo aleae. In: Hieronymi Cardani Opera omnia, t.1. Lugduni MDCLXIII. Sumptibus Ioannis Antonii Huguetan \& Marci Antonii Ravaud. Reprint 1966 Stuttgart: Frommann. - Englische Übersetzung in Ore, O. (1953): Cardano, The Gambling Scholar. Princeton N.J.: Princeton University Press.

[5] E. Coumet,(1970): La théorie du hasard est-elle née par hasard? Annales des Economies, Sociétés et Civilisations, 25, pp. 574-598.

[6] L. Daston, (1989): L'interprétation classique du Calcul des Probabilités. Annales des Economies, Sociétés et Civilisations 3, pp. 715-731. 
[7] Th. Deman, (1933): Probabilis. Revue des Sciences philosophiques et théologiques 22, pp. $260-290$.

[8] B. de Finetti, (1970): Teoria delle probabilità. Torino: Giulio Einaudi.

[9] G. Gigerenzer, Z. Swijtink, Th. Porter, L. Daston, J. Beatty, L. Krüger, (1989): The Empire of Chance. Cambridge: Cambridge University Press.

[10] I. Hacking,(1975) The emergence of Probability. London: Cambridge University Press

[11] J.L. Hodges, E.L. Lehmann, (1964): Basic Concepts of Probability and Statistics. San Francisco: Holden Day.

[12] Chr. Huygens, (1657) De ratiociniis in ludo aleae. In: Frans van Schooten, Exercitionum mathematicarum libri quinque, Liber V. Leiden: Ex officina Johannis Elsevirii. - Die holländische Fassung "Van Rekeningh in Spelen van Geluck" (1656, erschienen 1660) findet sich zusammen mit der französischen Übersetzung in: Oeuvres complètes de Chr. Huygens, t. XIV, La Haye 1920, Nijhoff. - Deutsche Übersetzung in Bernoulli, J. (1899).

[13] R. Ineichen, (1988): Dante - Kommentare und die Vorgeschichte der Stochastik. Historia Matematica 15, pp. 264-269.

[14] R. Ineichen, (1996): Würfel und Wahrscheinlichkeit - Stochastisches Denken in der Antike. Heidelberg, Berlin, Oxford: Spektrum Akadem. Verlag.

[15] A. de Moivre, (1738 bzw. 1756): The doctrine of chance, 3. Auflage. London. Millar. Reprint New York 1967: Chelsea. - Erste Auflage 1718 London: Pearson.

[16] P.R. de Montmort, (1708 bzw. 1713): Essay d'Analyse sur les jeux de hazard. Paris: Jacques Quillau. Reprint New York 1980: Chelsea.

[17] B. Pascal, (1654 bzw. 1954): Oeuvres complètes. Texte établi, présenté et annoté par J. Chevalier. Paris: Gallimard.

[18] F.P. Ramsey, (1931): The foundations of Mathematics and other logical essays. London: Kegan Paul, Trench, Trubner.

[19] Saint François de Sales, (1619 bzw. 1911): Introduction à la vie dévote. Nouvelle édition d'après celle de 1619. Annecy: J. Abry.

[20] S. Sambursky, (1965): Das physikalische Weltbild der Antike. Zürich: Artemis.

[21] I. Schneider, (1976): The introduction of probability into Mathematics. Historia Matematica 3, pp. 135140 .

[22] I. Schneider, (1979): Die Mathematisierung der Vorhersage künftiger Ereignisse in der Wahrscheinlichkeitstheorie vom 17. bis zum 19. Jahrhundert. Berichte zur Wissenschaftsgeschichte 2, pp. 101-112.

[23] I. Schneider, (1980): Why do we find the origin of a calculus of probability in the seventh century? In: J. Hintikka, D. Gruender and E. Agazzi (eds.); Pisa conference Proceedings II, pp. 3-24. Dordrecht: Reidel.

[24] I. Schneider, (1988): Die Entwicklung der Wahrscheinlichkeitstheorie von den Anfängen bis 1933 Einführungen und Texte. Darmstadt: Wissenschaftliche Buchgesellschaft.

[25] G. Shafer, (1978): Non-Additive Probabilities in the Work of Bernoulli and Lambert. Archive for History of Exact Sciences 19, pp. 309-370.

[26] O.B. Sheynin, (1974): On the Prehistory of the Theory of Probability. Archive for History of Exact Sciences 12, pp. 79-141.

Robert Ineichen

Institut de mathématiques

Université de Fribourg

CH-1700 Fribourg 